\title{
Results and future plans of the NEXT double beta decay experiment
}

\section{A. Laing*, On belhalf of the NEXT collaboration}

University of Texas at Arlington

E-mail: andrew. laingeuta.edu

The Neutrino Experiment with a Xenon TPC (NEXT) searches for the neutrinoless double beta decay of ${ }^{136} \mathrm{Xe}$ using a high pressure xenon gas time projection chamber. This detector technology has several key advantages, including excellent energy resolution, powerful event classification based on track topology, and favorable mass scalability. It also offers the tantalising possibility of tagging the daughter ion produced in the decay. The current stage of the experiment, NEXTWhite, has been been taking data at the Laboratorio Subterráneo de Canfranc (LSC) in Spain since late 2016.

In this talk, we will review recent results from NEXT-White after the first year of low-background operations using both xenon depleted in the ${ }^{136} \mathrm{Xe}$ isotope as a direct measure of background, and enriched xenon data to measure the two neutrino mode. Results form dedicated calibration runs to study detector performance will also be shown. Finally, we will conclude by discussing the experiment's prospects, starting from the NEXT-100 detector to be commissioned in 2020.

XXIX International Symposium on Lepton Photon Interactions at High Energies - LeptonPhoton2019 August 5-10, 2019

Toronto, Canada

${ }^{*}$ Speaker. 


\section{Introduction}

NEXT seeks to observe the $0 v \beta \beta$ decay of ${ }^{136} \mathrm{Xe}$ using a high pressure gas TPC. Amplification of the ionisation signal is achieved using a region of increased field at the end of the electron drift where secondary scintillation is induced in a process known as electroluminescence. In the current detectors two separate sensor groups are used to optimise the measurement of the energy of an event and to reconstruct the topology of the tracks left by ionising particles. This gives NEXT a rare handle in the rejection of backgrounds since the double electron indicative of a $\beta \beta$ decay results in a track with a region of high energy deposition at each end whereas the single elctrons induced by the Compton and photoelectric interactions of high energy gammas will result in a track with only one.

The technology was first demonstrated with $\mathrm{kg}$-scale detectors $[1,2]$ and is currently being exploited at a larger scale by the Next-White detector at Laboratorio Subterráneo de Canfranc (LSC) $[3,4,5]$. A rich scientific program is currently developing the next stage detector (Next-100) while looking to the future with multiple R\&D efforts for a tonne-scale NEXT. These proceedings give a general overview of the project.

\section{NEW results}

The Next-White (NEW) detector has been operating at Laboratorio Subterráneo de Canfranc (LSC) since the end of 2016. Calibration runs were taken over the course of 2017 with various radioactive sources leading to measurements of the expected energy resolution and the power of the topological reconstruction to identify double electron tracks. Figure 1 shows a fully reconstructed energy spectrum for runs with both ${ }^{137} \mathrm{Cs}$ and ${ }^{228} \mathrm{Th}$ sources. The main gamma peaks from the decay chains of these sources can be seen clearly. As reported in [4], a FWHM energy resolution of $\sim 0.91 \%$ is obtained at the energy of $Q_{\beta \beta}$. The same source data can be used to study the power

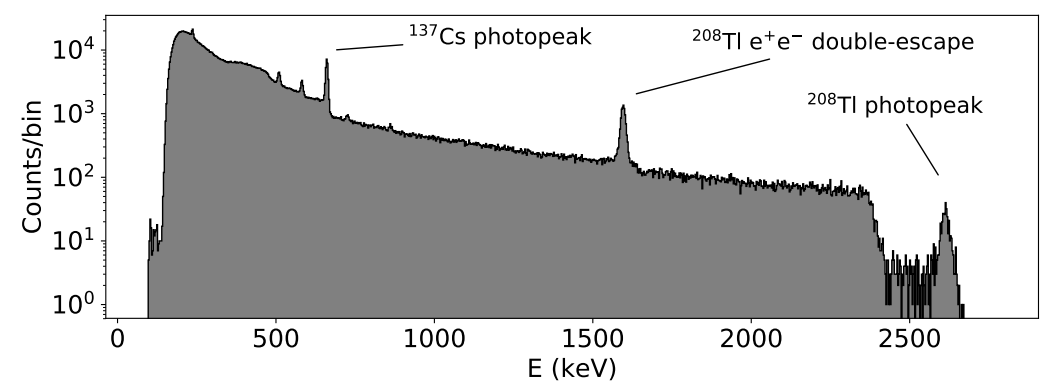

Figure 1: Reconstructed energy spectrum from events selected from interactions induced by ${ }^{137} \mathrm{Cs}$ and ${ }^{228}$ Th sources [4].

of topological reconstruction in NEXT. As mentioned in section 1, using the topology of the tracks NEXT is able to separate signal and background. The ${ }^{208} \mathrm{Th}$ gamma at $2.615 \mathrm{MeV}$ has enough energy for pair production interactions to be probable. In a pair production interaction, if the two annihilation gammas escape the active volume the track left in the detector is identical to those expected for $2 v \beta \beta$ events with energy $1.593 \mathrm{MeV}$. Treating these events as a model for signal it is 
possible to benchmark the topological rejection factors expected in a data-driven manner. In [3], the rejection power was shown to be in good agreement with the Monte Carlo expectations and to select $\sim 71 \%$ of signal-like events while rejecting $\sim 80 \%$ of the single electron tracks.

There are currently data from two main low-background runs on disk. At the end of 2018 data with xenon depleted in the ${ }^{136} \mathrm{Xe}$ isotope (RunIV) was used to benchmark the background model [5]. During 2019 data is being taken with the detector filled with xenon enriched to $\sim 91 \%$ in ${ }^{136} \mathrm{Xe}$. Analysis is ongoing with initial indications of a consistent measurement of the $2 v \beta \beta$ lifetime.

\section{NEXT-100}

The NEXT-100 detector is expected to start data runs towards the end of 2020. The detector will hold $\sim 100 \mathrm{~kg}$ of xenon at $15 \mathrm{bar}$ and will read the scintillation and make the energy measurement using 60 PMTs. Topological reconstruction will be made using an array of $>40001 \times 1 \mathrm{~mm}^{2}$ SiPMs. A technical drawing of the detector is shown in figure 2. Next-100 is a scaled up version

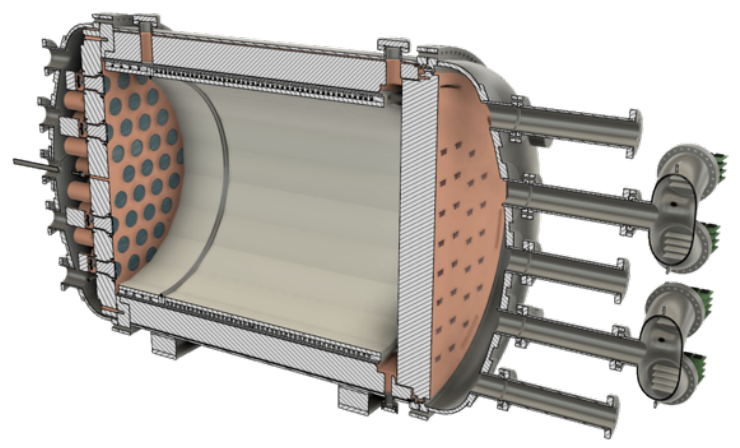

Figure 2: The NEXT-100 detector.

of NEW with certain improvements identified in NEW data taking. The gas flow will be improved over that achieved in NEW by increasing the number of entry points for the xenon to the active volume, SiPM base boards will be thinner to reduce the radioactive contribution of the kapton and glues and a muon tagging system will be added to improve cosmogenic identification. The detector is expected to have a sensitivity equal to or better than that reported in the original NEXT-100 sensitivity paper [6].

Multivariate and neural network analyses are also being developed to improve on the current simple cut based analysis used to calculate the background rejection factors and signal efficiences for $0 v \beta \beta$ [7].

\section{R\&D for tonne scale detectors}

The collaboration is already looking to advance the technology for detectors at scales of a metric tonne. While the design is relatively easy to scale with xenon being among the easiest isotopes to enrich, there are certain changes and improvements of interest when building a truely competitive detector. 
A multi-pronged approach sees R\&D effort towards a detector fully instrumented using SiPMs as a means to reduce radioactivity associated with the PMTs. Additionally, gas mixtures are being explored to improve diffusion and, therefore, topological reconstruction $[8,9,10]$. Elsewhere, $\mathrm{R} \& \mathrm{D}$ towards cooled gas operation as a means of reducing dark current in the SiPMs so that scintillation detection is still efficient.

$R \& D$ towards a feasible method of detecting the presence of ${ }^{136} \mathrm{Ba}$ ions in the gas is underway in a multidisciplinary effort to understand Single Molecule Fluorescence Imaging (SMFI) as a means to detect $\mathrm{Ba}^{++}$ions in dry media and the related machinery required for this method to be used in a TPC $[11,12,13]$.

\section{Summary}

The NEXT experiment is currently running its first underground detector with first measurements of the 2 neutrino half-life expected soon. The next stage of the experiment is being built and will be installed at LSC next year. Concurrent with these efforts multiple R\&D studies are underway in order to add to the advantages of this technology and achieve a virtually background-free experiment at the tonne scale.

\section{References}

[1] NEXT Collaboration, V. Alvarez et al., Operation and first results of the NEXT-DEMO prototype using a silicon photomultiplier tracking array, JINST 8 (2013) P09011, [arXiv: 1306.0471 ].

[2] NEXT Collaboration, P. Ferrario et al., First proof of topological signature in the high pressure xenon gas TPC with electroluminescence amplification for the NEXT experiment, JHEP 01 (2016) 104, [arXiv:1507.05902].

[3] NEXT Collaboration, P. Ferrario et al., Demonstration of the Event Identification Capabilities of the NEXT-White Detector, arXiv:1905.13141.

[4] NEXT Collaboration, J. Renner et al., Energy Calibration of the NEXT-White Detector with 1\% Resolution Near $Q_{\beta \beta}$ of ${ }^{136} \mathrm{Xe}$, arXiv:1905.13110.

[5] NEXT Collaboration, P. Novella et al., Radiogenic Backgrounds in the NEXT Double Beta Decay Experiment, arXiv:1905.13625.

[6] NEXT Collaboration, J. Martín-Albo et al., Sensitivity of NEXT-100 to Neutrinoless Double Beta Decay, JHEP 05 (2016) 159, [arXiv: 1511.09246$].$

[7] NEXT Collaboration, J. Renner et al., Background rejection in NEXT using deep neural networks, JINST 12 (2017), no. 01 T01004, [arXiv: 1609.06202 ].

[8] NEXT Collaboration, C. A. O. Henriques et al., Electroluminescence TPCs at the Thermal Diffusion Limit, JHEP 01 (2019) 027, [arXiv: 1806.05891$].$

[9] NEXT Collaboration, A. D. McDonald et al., Electron Drift and Longitudinal Diffusion in High Pressure Xenon-Helium Gas Mixtures, JINST 14 (2019), no. 08 P08009, [arXiv: 1902.05544 ].

[10] NEXT Collaboration, A. F. M. Fernandes et al., Electroluminescence Yield in low-diffusion Xe-He gas mixtures, arXiv:1906.03984. 
[11] A. D. McDonald et al., Demonstration of Single Barium Ion Sensitivity for Neutrinoless Double Beta Decay using Single Molecule Fluorescence Imaging, Phys. Rev. Lett. 120 (2018), no. 13132504 , [arXiv:1711.04782].

[12] P. Thapa, I. Arnquist, N. Byrnes, A. A. Denisenko, F. W. Foss, B. J. P. Jones, A. D. Mcdonald, D. R. Nygren, and K. Woodruff, Barium Chemosensors with Dry-Phase Fluorescence for Neutrinoless Double Beta Decay, arXiv:1904.05901.

[13] I. Rivilla et al., Towards a background-free neutrinoless double beta decay experiment based on a fluorescent bicolor sensor, arXiv:1909.02782. 